\title{
RESEARCH
}

Open Access

\section{Data-driven FDG-PET subtypes of Alzheimer's disease-related neurodegeneration}

Fedor Levin ${ }^{1}$, Daniel Ferreira ${ }^{2}$, Catharina Lange ${ }^{3,4}$, Martin Dyrba ${ }^{1}$, Eric Westman ${ }^{2,5}$, Ralph Buchert ${ }^{6}$, Stefan J. Teipel ${ }^{1,7}$, Michel J. Grothe ${ }^{1,8^{*}}$ (D) for the Alzheimer's Disease Neuroimaging Initiative

\begin{abstract}
Background: Previous research has described distinct subtypes of Alzheimer's disease (AD) based on the differences in regional patterns of brain atrophy on MRI. We conducted a data-driven exploration of distinct AD neurodegeneration subtypes using FDG-PET as a sensitive molecular imaging marker of neurodegenerative processes.

Methods: Hierarchical clustering of voxel-wise FDG-PET data from 177 amyloid-positive patients with AD dementia enrolled in the Alzheimer's Disease Neuroimaging Initiative (ADNI) was used to identify distinct hypometabolic subtypes of $A D$, which were then further characterized with respect to clinical and biomarker characteristics. We then classified FDG-PET scans of 217 amyloid-positive patients with mild cognitive impairment ("prodromal AD") according to the identified subtypes and studied their domain-specific cognitive trajectories and progression to dementia over a follow-up interval of up to 72 months.

Results: Three main hypometabolic subtypes were identified: (i) "typical" (48.6\%), showing a classic posterior temporoparietal hypometabolic pattern; (ii) "limbic-predominant" (44.6\%), characterized by old age and a memory-predominant cognitive profile; and (iii) a relatively rare "cortical-predominant" subtype (6.8\%) characterized by younger age and more severe executive dysfunction. Subtypes classified in the prodromal AD sample demonstrated similar subtype characteristics as in the AD dementia sample and further showed differential courses of cognitive decline.

(Continued on next page)
\end{abstract}

\footnotetext{
* Correspondence: michel.grothe@dzne.de

Data used in the preparation of this article were obtained from the Alzheimer's Disease Neuroimaging Initiative (ADNI) database (adni.loni.usc. edu). As such, the investigators within the ADNI contributed to the design and implementation of ADNI and/or provided data but did not participate in analysis or writing of this report. A complete listing of ADNI investigators can be found at http://adni.loni.usc.edu/wp-content/themes/freshnews-dev-v2/ documents/policy/ADNI_Acknowledgement_List\%205-29-18.pdf.

${ }^{1}$ German Center for Neurodegenerative Diseases (DZNE), Rostock/Greifswald, Rostock, Germany

${ }^{8}$ Unidad de Trastornos del Movimiento, Servicio de Neurología y Neurofisiología Clínica, Instituto de Biomedicina de Sevilla, Hospital Universitario Virgen del Rocío/CSIC/Universidad de Sevilla, Avda. Manuel Siurot, s/n, 41013 Sevilla, Spain

Full list of author information is available at the end of the article
}

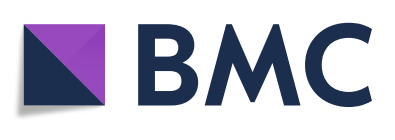

(c) The Author(s). 2021, corrected publication 2021. Open Access This article is licensed under a Creative Commons Attribution 4.0 International License, which permits use, sharing, adaptation, distribution and reproduction in any medium or format, as long as you give appropriate credit to the original author(s) and the source, provide a link to the Creative Commons licence, and indicate if changes were made. The images or other third party material in this article are included in the article's Creative Commons licence, unless indicated otherwise in a credit line to the material. If material is not included in the article's Creative Commons licence and your intended use is not permitted by statutory regulation or exceeds the permitted use, you will need to obtain permission directly from the copyright holder. To view a copy of this licence, visit http://creativecommons.org/ licenses/by/4.0/. The Creative Commons Public Domain Dedication waiver (http://creativecommons.org/publicdomain/zero/1. 0/) applies to the data made available in this article, unless otherwise stated in a credit line to the data. 
(Continued from previous page)

Conclusions: These findings complement recent research efforts on MRI-based identification of distinct AD atrophy subtypes and may provide a potentially more sensitive molecular imaging tool for early detection and characterization of AD-related neurodegeneration variants at prodromal disease stages.

Keywords: Alzheimer's disease, Subtypes, Mild cognitive impairment, Prodromal, FDG-PET, Hypometabolism

\section{Introduction}

Previous research has demonstrated heterogeneity in Alzheimer's disease (AD) which is linked to distinct neuropathological subtypes of AD characterized by limbic-predominant, hippocampal sparing, or rather balanced ("typical") spatial distributions of neurofibrillary tangle pathology [1]. Analysis of ante-mortem structural MRI data demonstrated that neuropathologically defined AD subtypes also show characteristic in vivo patterns of regional brain atrophy [2]. Recent research has used clustering methods on structural MRI data to identify similar regional atrophy subtypes in AD in a data-driven manner [3]. Interestingly, these atrophy subtypes could already be detected in patients with prodromal AD (i.e. amyloid-beta $[A \beta]$-positive patients with mild cognitive impairment $[\mathrm{MCI}]$ ), who showed similar biomarker characteristics as the subtypes identified in patients with $\mathrm{AD}$ dementia and were associated with differential clinical trajectories $[4,5]$.

In addition to volumetric information from structural MRI, hypometabolism in FDG-PET is a well-established imaging marker of neurodegeneration as recognized by the recently revised research criteria for $\mathrm{AD}$ [6]. The use of FDG-PET in dementia and neurodegenerative cognitive impairment was also recently supported by consensus recommendations from a panel of experts from the European Association of Nuclear Medicine and the European Academy of Neurology [7]. FDG-PET may indicate a decrease in cerebral glucose metabolism that occurs prior to the macroscopic atrophy detectable with MRI, rendering the technique potentially more sensitive to early neurodegenerative processes [8-10]. While previous hypothesis-driven studies have also reported differential hypometabolic FDG-PET patterns amongst AD dementia patients [11-13], to our knowledge, FDGPET has not yet been used for identifying neurodegeneration subtypes of $\mathrm{AD}$ in a data-driven manner. Given that detection of an "AD-typical" hypometabolism pattern by visual inspection of individual patients' FDGPET scans is commonly used for differential dementia diagnosis in research and clinical settings [7, 12, 14-16], a systematic evaluation of whether and how specific AD neurodegeneration subtypes are reflected in FDG-PET data can have important diagnostic implications.

In the current study, we investigated hypometabolic subtypes in patients with AD by applying an established hierarchical clustering approach to a large dataset of FDG-PET scans from patients with biomarker-confirmed AD enrolled in the Alzheimer's Disease Neuroimaging Initiative (ADNI). The identified subtypes were further characterized with respect to detailed clinical and biomarker profiles and also used for subtype stratification of an independent ADNI sample of patients with prodromal $\mathrm{AD}$ (A $\beta$-positive patients with $\mathrm{MCI}$ ) who were clinically followed for up to 72 months.

\section{Methods \\ Participants}

We included data from 179 cognitively normal (CN) participants (58 A $\beta$-positive), $177 \mathrm{~A} \beta$-positive patients with a clinical diagnosis of $\mathrm{AD}$ dementia, and $217 \mathrm{~A} \beta$ positive patients with $\mathrm{MCI}$ (i.e. prodromal $\mathrm{AD}$ ) from the ADNI-1, ADNI-GO/2 and ADNI-3 cohorts (adni.loni. usc.edu). The detailed inclusion criteria for the different diagnostic categories have been described in detail before [17] and are available on the ADNI website (http:// adni.loni.usc.edu/methods/documents/). Evidence of $A \beta$ pathology was based on AV45-PET or, in case this measure was not available, on CSF A $\beta$ levels (see below for details). This was only the case for $48 \mathrm{AD}$ dementia patients (i.e. $12 \%$ of the total patient sample). The ADNI is a longitudinal multicentre study aimed at investigating whether neuroimaging methods such as MRI and PET, together with genetic, clinical and neuropsychological measures, can be used to characterize the progression of MCI and AD. The ADNI was launched in 2003 as a public-private partnership, led by principal investigator Michael W. Weiner, MD.

\section{Neuropsychological test scores}

Cognitive performance in the ADNI is assessed using neuropsychological test batteries covering various cognitive domains. We used previously established composite cognitive scores for memory (ADNI-MEM), executive function (ADNI-EF), visuospatial function (ADNI-VS) and language (ADNI-Lan) [18-20]. Mini-Mental State Examination (MMSE) scores were used for characterizing global cognitive impairment. We also calculated the difference between the ADNI-MEM and the ADNI-EF composite scores (ADNI-DIFF) to characterize differential decline in these two domains. Positive values in this variable thus represent a more pronounced executive 
impairment compared to the memory deficit, and vice versa for negative values.

\section{Longitudinal analysis}

We analysed longitudinal changes in cognitive functions of the prodromal $\mathrm{AD}$ subtypes for participants with available follow-up data $(n=200)$. We used longitudinal measures of ADNI-MEM, ADNI-EF, ADNI-VS and ADNI-Lan. Additionally, longitudinal Clinical Dementia Rating (CDR) scores were used as a criterion indicating a progression from prodromal $(C D R=0.5)$ to clinically manifest $A D$ dementia $(C D R \geq 1)$. The mean follow-up period was 44 months (range 12-72 months), and $72.5 \%$ of participants had at least 36 months of follow-up available.

\section{Biomarkers}

Measures of cortex-to-whole cerebellum AV45 standard uptake value ratios (SUVR) have been calculated by the ADNI PET core (Jagust Lab, UC Berkeley) and were downloaded from the ADNI server. We selected A $\beta$ positive patients with AD or MCI if their AV45 SUVR values were greater than or equal to the recommended threshold of 1.11 [21].

ADNI CSF values in the current study were derived from electrochemiluminescence immunoassays for $A \beta$ [1-42], tau phosphorylated at threonine 181 (p-tau) and total tau (t-tau) on an automated Elecsys cobas e 601 instrument. ${ }^{1}$ We included participants who had CSF A $\beta$ values lower than the threshold of $880 \mathrm{pg} / \mathrm{ml}$ proposed by Hansson et al. [22].

Selecting patients based on biomarker evidence of amyloidosis follows the design of previous MRI-based subtyping studies of differential neurodegeneration patterns in $\mathrm{AD}[4,5]$. However, given that the 2018 NIA-AA revised research criteria now reserve the term "Alzheimer's disease" for combined evidence of amyloid and tau positivity [6], we also categorized patients with regard to the full $\mathrm{A} / \mathrm{T} / \mathrm{(N)}$ classification using CSF p-tau levels above $19.2 \mathrm{pg} / \mathrm{ml}$ for denoting tau biomarker $(\mathrm{T})$ positivity and CSF t-tau levels above $242 \mathrm{pg} / \mathrm{ml}$ for denoting neurodegeneration biomarker $(\mathrm{N})$ positivity [23]. Full CSF biomarker data was available for $93 \%$ of our study sample (Supplementary Table 1).

$A P O E$ genotype was determined using DNA extracted from a 3-ml aliquot of EDTA blood samples by Cogenics

\footnotetext{
${ }^{1}$ The Elecsys $\beta$-Amyloid [1-42] CSF immunoassay in use is not a commercially available IVD assay. It is an assay that is currently under development and for investigational use only. The measuring range of assay is 200 (lower technical limit) - $1700 \mathrm{pg} / \mathrm{ml}$ (upper technical limit). The performance of the assay beyond the upper technical limit has not been formally established. Therefore, the use of values above the upper technical limit, which are provided based on an extrapolation of the calibration curve, is restricted to exploratory research purposes and is excluded for clinical decision making or for the derivation of medical decision points.
}

[24]. Genotype information was coded in a binary $A P O E$ $\varepsilon 4$ variable indicating the presence of at least one $A P O E$ $\varepsilon 4$ allele.

Structural MRI images were used to derive hippocampal and cortical volume measures. MRI images in ADNI are acquired at multiple sites using scanner-specific T1weighted sagittal 3D MPRAGE sequences and undergo standardized image pre-processing steps to improve uniformity across the scanners (http://adni.loni.usc.edu/ methods/documents/). We extracted regional grey matter volumes from these scans using a previously described automated volumetry approach implemented in statistical parametric mapping software (SPM8, Wellcome Trust Center for Neuroimaging) and the VBM8-toolbox [25, 26]. Briefly, this involves automated tissue class segmentation and high-dimensional spatial normalization to an ageing/AD-specific reference template. Spatially normalized grey matter (GM) maps were visually inspected for segmentation and normalization accuracy, and voxel values were modulated for volumetric changes introduced by the high-dimensional normalization, so that the total GM volume present before warping was preserved. Hippocampal (HV) and regional cortical grey matter volumes were extracted from these scans using regions-ofinterest defined in the Harvard-Oxford anatomical atlas [27]. Individual volumes were divided by the total intracranial volume (TIV), calculated as the sum of total volumes of all tissue segments. In analogy to previous MRI-based subtyping studies $[2,28]$, cortical grey matter volumes were extracted from selected frontal, temporal and parietal association areas (see Supplementary Table 2), summed into a measure of bilateral cortical total volume (CTV), and further used to calculate the hippocampal to cortical volume ratio (HV:CTV).

Finally, we included white matter hyperintensity (WMH) volume as a measure of small vessel vascular disease burden. These values have been calculated by the ADNI MRI core and were downloaded from the ADNI server. In ADNI-1 data, WMH values were obtained via analysis of the proton density (PD) and T1 and T2 MRIs [29]. In ADNI-GO/2, a fluid-attenuated inversion recovery (FLAIR) MRI sequence was used to calculate WMH volumes [30]. In the present study, we pooled available WMH measures [4] and controlled statistical analyses of this variable for different segmentation methods using a dummy-coded confound variable.

\section{FDG-PET data acquisition and preprocessing}

FDG-PET data were retrieved in a pre-processed form from the ADNI server. FDG-PET images were obtained on multiple scanners with protocols specific to platforms. Dynamic 3D scans of six 5-min frames were acquired $30-60 \mathrm{~min}$ after injections of $185 \mathrm{MBq}$ of ${ }^{18} \mathrm{~F}$ FDG. All original ADNI FDG-PET scans underwent 
standardized image pre-processing steps to improve uniformity across the scanners. Detailed information on FDG-PET acquisition and pre-processing is available on the ADNI website (http://adni.loni.usc.edu/methods/ documents/). For the present study, FDG-PET images were further spatially normalized to a customized FDGPET standard space template and smoothed with a Gaussian smoothing kernel of $8 \mathrm{~mm}$ full-width at half maximum (FWHM) using SPM8 [31].

\section{Hierarchical clustering}

The patients with $\mathrm{AD}$ dementia were classified into hypometabolic subtypes using agglomerative hierarchical clustering of voxel-wise FDG-PET data with Ward's linkage as implemented in the MATLAB software [32, 33]. Individual FDG-PET profiles were scaled to their global mean prior to clustering analysis so that clustering relied on the differences in regional hypometabolic patterns rather than on potential differences in global hypometabolism severity across patients [32]. Global intensity scaling is a commonly used approach in neuroimaging-based subtyping studies to control clustering analyses for individual differences in disease severity (see [3] for a recent review of this literature). Accordingly, similar global scaling methods were also used in previous FDG-PET studies that employed hierarchical clustering approaches for data-driven characterizations of FDG-PET subtypes in other neurodegenerative dementias [32, 34].

In the clustering procedure, the algorithm progressively combines closest voxel-wise FDG-PET profiles of the participants into larger clusters, as well as most similar clusters with each other. The output of the algorithm is a hierarchical dendrogram in which the level of branching indicates the degree of dissimilarity between the clusters. The optimal number of separable clusters in the data was evaluated using standard performance measures for clustering solutions including the DaviesBouldin criterion [35] and the silhouette criterion [36].

To visualize the patterns of hypometabolism in the identified subtypes, we conducted voxel-wise twosample $t$ tests between FDG-PET images from each of the subtypes and the $\mathrm{CN}$ group, using age, gender and years of education as covariates. In contrast to the global signal scaling used in the clustering procedure, images were scaled to the average signal in a pons reference region for this analysis as the standard method to assess hypometabolism in comparison with a healthy control group [31]. Global signal scaling is typically not recommended in this context, because it accounts for global disease-related differences in glucose utilization that typically exist between dementia patients and healthy controls and thus lowers the sensitivity to detect regional hypometabolism [37, 38]. An explicit grey matter mask was applied to the images, and obtained $t$ values were converted into Cohen's $d$ effect size values. Analogous voxel-wise analyses were also conducted to directly compare the different subtypes identified in the AD group.

\section{Classification of patients with prodromal AD}

We classified FDG-PET scans of patients with prodromal $\mathrm{AD}$ according to the identified $\mathrm{AD}$ subtypes using a fully automated classification procedure. For that, we first screened patients for evidence of regional hypometabolism by assessing whether at least one of the 48 bilateral cortical areas defined in the Harvard-Oxford atlas had an FDG-PET signal (scaled to pons) of at least one standard deviation below the mean of the control group. Participants with no such regions were classified into a "no hypometabolism" subtype $[12,14]$. The remaining patients with prodromal $\mathrm{AD}$ were classified into one of the subtypes identified in the AD dementia group based on the smallest Euclidean distance between the individual patient's voxel-wise FDG-PET profile (scaled to global values) and the mean FDG-PET profile of each of the AD dementia subtypes [4]. Global scaling was used for this classification procedure in order to match the individual FDG patterns of the MCI patients to the regional patterns defined by the $\mathrm{AD}$ subtypes independent of global hypometabolism severity. Visualization of the hypometabolism patterns of the classified subtypes as compared to the $\mathrm{CN}$ group employed identical voxel-wise two-sample $t$ tests of pons-scaled FDG-PET images as described above.

\section{Statistical analysis}

Statistical analyses were conducted using RStudio and R version 3.5.2 with a statistical significance threshold of $P<0.05$ (two-tailed). Chi-squared tests with post hoc pairwise proportion tests were used to compare gender compositions of subtypes and frequencies of the $A P O E$ $\varepsilon 4$ genotype. For this and other post hoc tests comparing the subtypes, we used the false discovery rate (FDR) correction [39] as implemented in R. Age and years of education were compared across subtypes using ANOVA. Differences in cognitive measures and biomarkers across the subtypes were tested with ANCOVA using age, gender and education as covariates.

For patients with prodromal AD with available clinical follow-up data, we also conducted Cox proportional hazards regression analyses for analysing differential risks of progression to dementia across FDG-PET defined subtypes. Progression to dementia was operationalized as a change in CDR score from 0.5 to $\geq 1$ [40]. Models included age, gender and education as covariates. Participants were censored if they did not progress to dementia before the last available follow-up CDR score.

In addition, linear mixed effects regression models were used to assess the differences in domain-specific 
longitudinal changes in memory, executive function, visuospatial function or language function [40, 41]. Models included the time of follow-up measured in months from baseline, a factor variable indicating subtype and an interaction term for the time by subtype as independent variables. The estimates for interactions between subtype and time indicated whether subtypes had differential cognitive trajectories over time. Age, gender and education were included as covariates. Regression models included random intercepts and random slopes for participants; $t$ tests used Satterthwaite approximations for degrees of freedom.

\section{Results}

Identification of hypometabolic subtypes in patients with AD dementia

We used objective criteria for evaluating optimal clustering solutions to select the level of cutoff for the hierarchical clustering dendrogram supported by the data. The Davies-Bouldin criterion favoured solutions with three or five clusters, whereas the silhouette criterion favoured three clusters (see Supplementary Figure 1). Therefore, we chose the clustering solution with three clusters (see results for solutions with higher cluster numbers in the Supplementary Figure 2). Cluster-1 included $44.6 \%$ of the patients and showed a predominantly limbic hypometabolic profile with most pronounced hypometabolism in the medial temporal lobe, which extended to the posterior cingulate cortex, lateral temporo-parietal areas and also large areas of the ventromedial and lateral frontal lobe (Fig. 1, henceforth referred to as the "limbicpredominant" subtype). Cluster- 2 included $48.6 \%$ of the patients and corresponded to a more AD-typical pattern of marked posterior temporo-parietal hypometabolism with additional, albeit less pronounced, involvement of the medial temporal lobe (henceforth referred to as the "typical" subtype). Cluster-3 included $6.8 \%$ of the patients and showed a pattern of temporo-parietal cortical hypometabolism similar to that of cluster-2, but with more extensive involvement of the frontal lobe and largely spared metabolism in the medial temporal lobe (henceforth referred to as the "cortical-predominant" subtype). Direct comparisons of regional hypometabolism between subtypes are shown in Supplementary Figure 3.

\section{Clinical and biological characterization of the hypometabolic $A D$ dementia subtypes}

Demographic, clinical and biomarker characteristics of the three AD subtypes are listed in Table 1. The "limbicpredominant" hypometabolic subtype was the oldest with an average age of 75.4 years and the "cortical-

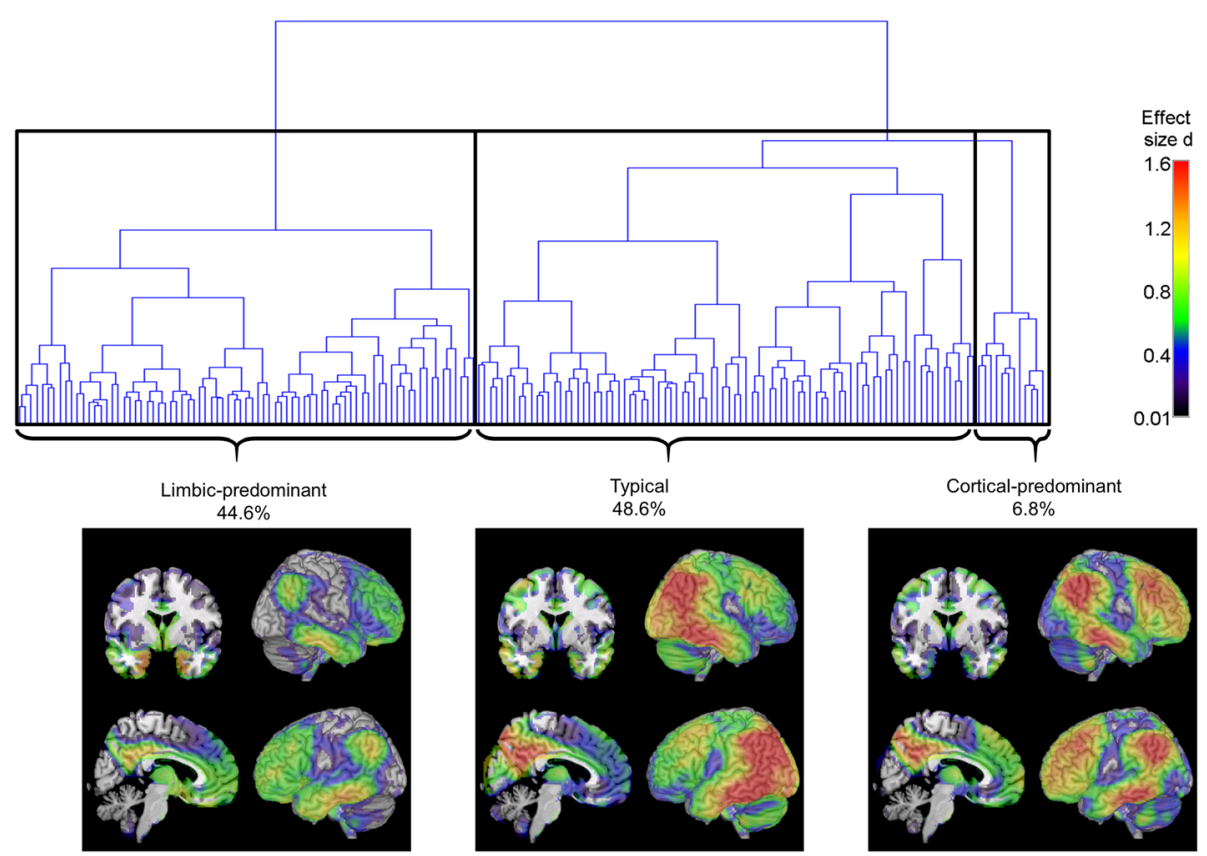

Fig. 1 Hierarchical clustering dendrogram and hypometabolic FDG-PET patterns of identified AD subtypes. Dendrogram resulting from Ward's hierarchical clustering analysis of individual FDG-PET profiles of patients with AD dementia. Brain plots show voxel-wise hypometabolic patterns of the three identified AD subtypes as revealed by statistical comparison to the healthy control group. FDG-PET scans were scaled to the average pons signal prior to the group comparisons, and age, gender, and years of education were used as covariates. Statistical parametric maps of the group differences were converted into Cohen's $d$ effect size maps to allow for a better comparison of the patterns across the unevenly sized AD subgroups. Subtype patterns at higher clustering solutions are shown in Supplementary Figure 2 
Table 1 Demographic, clinical and biomarker characteristics of AD dementia subtypes at baseline

\begin{tabular}{|c|c|c|c|c|c|c|c|c|}
\hline & \multirow[t]{2}{*}{ CN group } & \multirow{2}{*}{$\begin{array}{l}\text { AD group, limbic- } \\
\text { predominant (S1) }\end{array}$} & \multirow{2}{*}{$\begin{array}{l}\text { AD group, } \\
\text { typical (S2) }\end{array}$} & \multirow{2}{*}{$\begin{array}{l}\text { AD group, cortical- } \\
\text { predominant }(\mathrm{S} 3)\end{array}$} & \multirow{2}{*}{$\begin{array}{l}P \text { value, global } \\
\text { comparison } \\
\text { (S1, S2 and S3) }\end{array}$} & \multicolumn{3}{|c|}{ Pair-wise comparisons } \\
\hline & & & & & & S1 vs S2 & S1 vs S3 & S2 vs S3 \\
\hline \multicolumn{9}{|l|}{ Demographics } \\
\hline$n(\%)$ & 179 & 79 (44.6\%) & $86(48.6 \%)$ & $12(6.8 \%)$ & & & & \\
\hline Age, years & $73.8(6.5)$ & $75.4(6.9)$ & $73.2(5.7)$ & $68.0(7.7)$ & 0.007 & 0.149 & 0.015 & 0.088 \\
\hline Sex, female (\%) & $50 \%$ & $49 \%$ & $38 \%$ & $50 \%$ & 0.332 & & & \\
\hline Education, years & $16.6(2.5)$ & $15.4(3.1)$ & $15.5(2.6)$ & $16.3(2.6)$ & 0.544 & & & \\
\hline \multicolumn{9}{|l|}{ Cognition } \\
\hline MMSE & $29.1(1.2)$ & $23.4(1.9)$ & $23.2(2.2)$ & $22.0(2.2)$ & 0.037 & 0.798 & 0.165 & 0.200 \\
\hline ADNI-MEM & $1.04(0.62)$ & $-0.85(0.55)$ & $-0.90(0.49)$ & $-1.31(0.44)$ & 0.012 & 1 & 0.025 & 0.028 \\
\hline ADNI-EF & $0.92(0.83)$ & $-0.65(0.86)$ & $-1.11(0.89)$ & $-1.73(0.81)$ & $<0.001$ & 0.002 & $<0.001$ & 0.043 \\
\hline ADNI-DIFF & $0.13(0.72)$ & $-0.21(0.69)$ & $0.21(0.85)$ & $0.41(0.74)$ & $<0.001$ & 0.004 & 0.029 & 0.723 \\
\hline ADNI-VS & $0.23(0.59)$ & $-0.42(0.8)$ & $-0.67(1.01)$ & $-1.15(1.08)$ & 0.085 & & & \\
\hline ADNI-Lan & $0.89(0.71)$ & $-0.63(0.95)$ & $-0.83(0.88)$ & $-1.11(0.78)$ & 0.03 & 0.406 & 0.406 & 0.588 \\
\hline \multicolumn{9}{|l|}{ Biomarkers } \\
\hline APOE $\varepsilon 4(\%)$ & $28 \%$ & $81 \%$ & $79 \%$ & $58 \%$ & 0.222 & & & \\
\hline AV45-PET SUVR & $1.11(0.18)$ & $1.43(0.14)$ & $1.47(0.17)$ & $1.4(0.17)$ & 0.145 & & & \\
\hline CSF $A \beta, p g / m l$ & 1392 (663) & $598(163)$ & $585(225)$ & 629 (169) & 0.686 & & & \\
\hline CSF t-tau, pg/ml & $236(92)$ & $374(143)$ & 374 (154) & $402(124)$ & 0.978 & & & \\
\hline CSF p-tau, pg/ml & $22(9)$ & $38(16)$ & $37(16)$ & $39(14)$ & 0.881 & & & \\
\hline $\mathrm{HV}$ & $4.97(0.38)$ & $4.05(0.51)$ & $4.1(0.38)$ & $4.46(0.46)$ & 0.09 & & & \\
\hline CTV & $87.39(6.28)$ & 75.84 (6.95) & 73.99 (6.23) & $70.04(5.88)$ & 0.006 & 0.136 & 0.026 & 0.136 \\
\hline HV:CTV ratio & $57.11(5.29)$ & $53.67(7.57)$ & $55.74(6.61)$ & $64.29(10.08)$ & $<0.001$ & 0.136 & $<0.001$ & $<0.001$ \\
\hline WMH & $6.1(10.4)$ & $8.0(10.0)$ & $5.8(7.9)$ & $8.1(12.7)$ & 0.262 & & & \\
\hline
\end{tabular}

Values for variables are presented as percentages (for gender and APOE $\varepsilon 4$ genotype) or means with standard deviation in parentheses. Missing values are excluded (for numbers of missing values per subtype see Supplementary Table 8). In case of significant main effects, subtypes were compared with the post hoc pairwise $t$ tests with FDR correction. Please note that composite cognitive scores have arbitrary units on scales centred on the original test samples used to develop these scores (including patients and healthy controls) [18-20]

S1 limbic-predominant subtype, S2 typical subtype, S3 cortical-predominant subtype, HV hippocampal grey matter volume scaled to total intracranial volume, CTV cortical composite grey matter volume scaled to total intracranial volume

predominant" subtype the youngest with an average age of 68.0 years. The "limbic-predominant" subtype generally showed the least pronounced impairments in neuropsychological testing and was characterized by a memory-predominant cognitive profile, whereas in the "typical" and especially the "cortical-predominant" subtype, the impairment in executive functions exceeded the mnestic deficit. Reflecting the subtype-defining hypometabolic patterns, hippocampal volume was lowest in the "limbic-predominant" subtype and highest in the "cortical-predominant" subtype, whereas cortical volumes showed the opposite behaviour. The resulting HV: CTV ratio was significantly higher for the "cortical-predominant" subtype in comparison with the two other subtypes. The "cortical-predominant" subtype also had a strikingly lower percentage of $A P O E \varepsilon 4$ carriers compared to the other two subtypes, although this difference did not reach statistical significance. No differences were observed between the subtypes with regard to gender distribution, years of education, molecular biomarkers of $\mathrm{A} \beta$ and tau pathology burden, or WMH volume (Table 1).

\section{Stratification of prodromal AD patients by hypometabolic subtypes}

About a quarter (26.3\%) of the prodromal AD cohort did not show any evidence of regional hypometabolism and was thus classified as "no hypometabolism" subtype. Amongst the other patients with prodromal AD, 49.8\% were classified into the "limbic-predominant" subtype, $22.6 \%$ into the "typical" subtype and only three participants $(1.4 \%)$ were classified into the "cortical-predominant" subtype, which was thus omitted from further analyses. Hypometabolic patterns of the classified subtypes in the prodromal AD cohort showed a strong spatial resemblance with the subtype-defining patterns in the AD dementia cohort (Fig. 2), thus corroborating the validity of the automated classification procedure. 


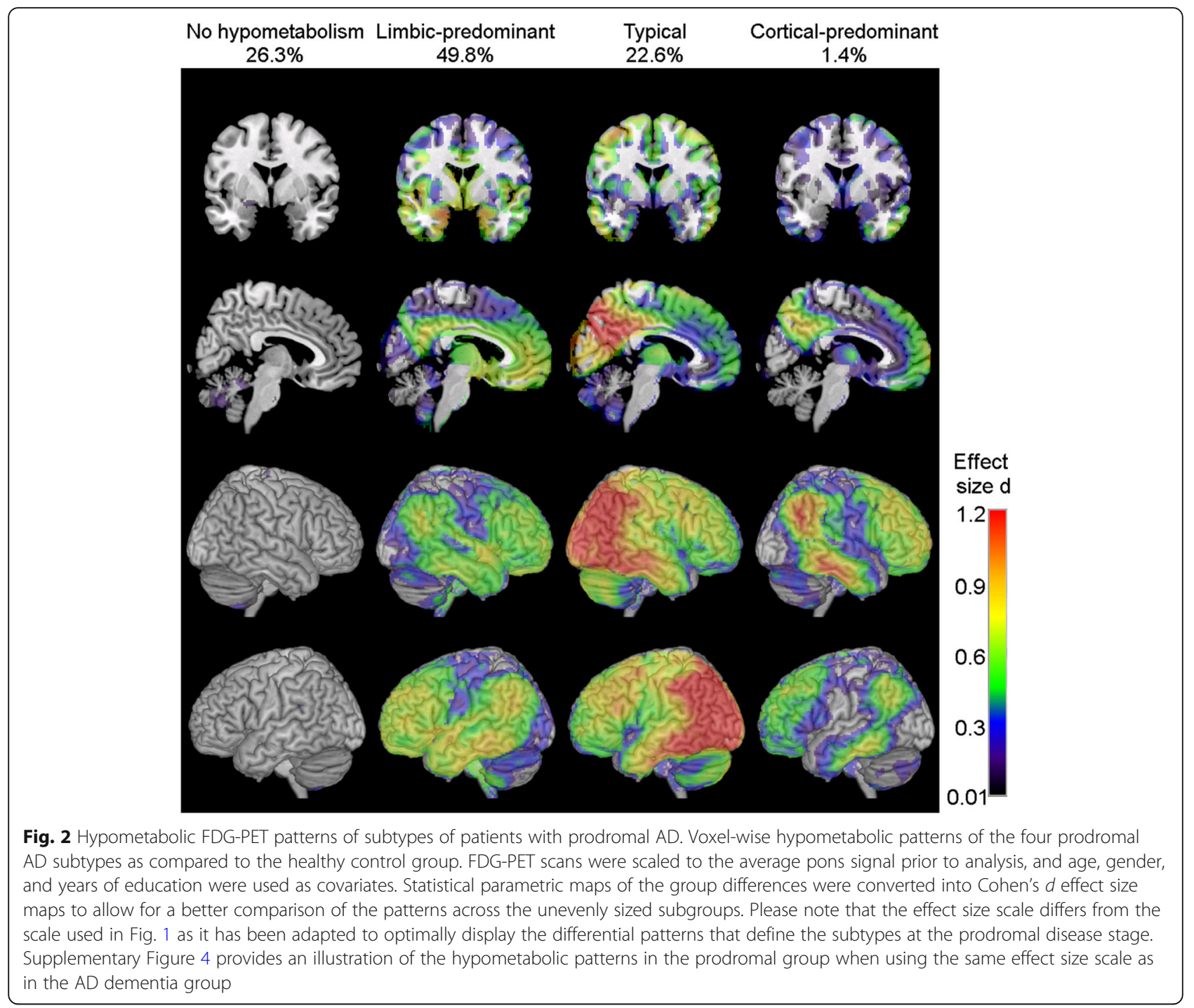

Demographic, clinical and biomarker characteristics of the subtypes are summarized in Table 2. The "limbicpredominant" subtype was again characterized by a significantly higher age compared to the other subtypes. The "no hypometabolism" subtype was the youngest and also had a higher proportion of females. Despite no pairwise differences in MMSE scores, the "no hypometabolism" subtype also showed significantly better memory and executive function performance than the "typical" and the "limbic-predominant" subtypes. Although we selected only A $\beta$-positive patients, the "no hypometabolism" subtype showed a significantly lower $A \beta$ burden on both PET and CSF measures. Nevertheless, patients in the "no hypometabolism" subtype showed a comparably high proportion of concomitant tau biomarker positivity as the other subtypes in the prodromal AD group (8690\%; Supplementary Table 1). In accordance with the
FDG-PET characteristics, the "no hypometabolism" subtype had significantly higher hippocampal and cortical volumes compared to the "limbic-predominant" and the "typical" subtypes.

Analysis of longitudinal clinical data showed that the "no hypometabolism" subtype was at the lowest risk of progression to dementia (vs "limbic-predominant": HR, 4.82, $P<0.001$; vs "typical": HR, 5.99, $P<0.001$; Fig. 3) and also showed a significantly slower decline in all four cognitive domains compared to the "typical" and the "limbic-predominant" subtypes (Fig. 4, see Supplementary Tables 3 and 4 for full model stats). Interestingly, the "typical" and "limbic-predominant" subtypes showed a similar decrease in memory function over time, but the "typical" subtype showed a significantly faster decline in executive function $(t=-2.25, P=0.026)$ and a trend towards 
Table 2 Demographic, clinical and biomarker characteristics of prodromal AD subtypes at baseline

\begin{tabular}{|c|c|c|c|c|c|c|c|}
\hline & \multirow{2}{*}{$\begin{array}{l}\text { Prodromal AD } \\
\text { group, no } \\
\text { hypometabolism (S0) }\end{array}$} & \multirow{2}{*}{$\begin{array}{l}\text { Prodromal AD group, } \\
\text { limbic-predominant (S1) }\end{array}$} & \multirow{2}{*}{$\begin{array}{l}\text { Prodromal } \\
\text { AD group, } \\
\text { typical (S2) }\end{array}$} & \multirow{2}{*}{$\begin{array}{l}P \text { value, global } \\
\text { comparison } \\
\text { (S0, S1 and S2) }\end{array}$} & \multicolumn{3}{|c|}{ Pair-wise comparisons } \\
\hline & & & & & S0 vs S1 & S0 vs S2 & S1 vs S2 \\
\hline \multicolumn{8}{|l|}{ Demographics } \\
\hline$n(\%)$ & $57(26.3 \%)$ & $108(49.8 \%)$ & $49(22.6 \%)$ & & & & \\
\hline Age, years & $68.4(6.6)$ & $76.1(5.7)$ & $71.7(6.2)$ & $<0.001$ & $<0.001$ & 0.009 & $<0.001$ \\
\hline Sex, female (\%) & $60 \%$ & $35 \%$ & $41 \%$ & 0.01 & 0.024 & 0.226 & 1 \\
\hline Education, years & $16.2(2.8)$ & $15.7(3.0)$ & $16.4(2.6)$ & 0.344 & & & \\
\hline \multicolumn{8}{|l|}{ Cognition } \\
\hline MMSE & $28.2(1.8)$ & $27.6(1.8)$ & $27.3(1.8)$ & 0.053 & & & \\
\hline ADNI-MEM & $0.57(0.63)$ & $0.05(0.61)$ & $-0.06(0.65)$ & $<0.001$ & $<0.001$ & $<0.001$ & 0.602 \\
\hline ADNI-EF & $0.76(0.91)$ & $0.00(0.77)$ & $0.15(1.01)$ & 0.018 & $<0.001$ & 0.001 & 0.542 \\
\hline ADNI-DIFF & $-0.19(0.83)$ & $0.05(0.78)$ & $-0.21(0.77)$ & 0.616 & & & \\
\hline ADNI-VS & $0.08(0.65)$ & $-0.06(0.76)$ & $-0.21(0.67)$ & 0.135 & & & \\
\hline ADNI-Lan & $0.6(0.7)$ & $-0.05(0.74)$ & $0.29(0.79)$ & 0.007 & $<0.001$ & 0.062 & 0.025 \\
\hline \multicolumn{8}{|l|}{ Biomarkers } \\
\hline APOE $\varepsilon 4(\%)$ & $68 \%$ & $62 \%$ & $77 \%$ & 0.163 & & & \\
\hline AV45-PET SUVR & $1.31(0.17)$ & $1.39(0.17)$ & $1.43(0.15)$ & 0.004 & 0.015 & 0.002 & 0.238 \\
\hline CSF A $\beta, p g / m l$ & $921(437)$ & 736 (237) & $672(214)$ & 0.002 & $<0.001$ & $<0.001$ & 0.417 \\
\hline CSF t-tau, pg/ml & 315 (134) & 337 (137) & 357 (144) & 0.123 & & & \\
\hline CSF p-tau, pg/ml & $31(15)$ & $34(16)$ & $37(16)$ & 0.096 & & & \\
\hline $\mathrm{HV}$ & $4.86(0.45)$ & $4.53(0.51)$ & $4.57(0.41)$ & 0.009 & $<0.001$ & 0.005 & 1 \\
\hline CTV & 89.87 (5.45) & 83.19 (6.48) & $82.93(5.35)$ & $<0.001$ & $<0.001$ & $<0.001$ & 1 \\
\hline HV:CTV ratio & $54.26(6.07)$ & $54.57(6.27)$ & $55.24(5.56)$ & 0.344 & & & \\
\hline WMH & $4.9(5.0)$ & $11.3(12.8)$ & $6.8(6.5)$ & 0.172 & & & \\
\hline
\end{tabular}

Cortical-predominant subtype of prodromal AD group $(n=3)$ not included. Values for variables are presented as percentages (for gender and APOE $\varepsilon 4$ genotype) or means with standard deviation in parentheses. Missing values are excluded (for numbers of missing values per subtype, see Supplementary Table 8). In case of significant main effects, subtypes were compared with the post hoc pairwise $t$ tests with FDR correction. Please note that composite cognitive scores have arbitrary units on scales centred on the original test samples used to develop these scores (including patients and healthy controls) [18-20]

SO no hypometabolism subtype, S1 limbic-predominant subtype, S2 typical subtype, HV hippocampal grey matter volume scaled to total intracranial volume, CTV cortical composite grey matter volume scaled to total intracranial volume

faster decline in visuospatial functions $(t=-1.931$, $P=0.055$; see Supplementary Table 5 for full model stats).

\section{Discussion}

In the current study, we conducted the first data-driven characterization of systematic heterogeneity in individuallevel FDG-PET patterns amongst AD dementia patients, and provide evidence for the existence of three distinct hypometabolic subtypes of $\mathrm{AD}$. These subtypes include a "typical" subtype of posterior temporo-parietal hypometabolism, as well as distinct "limbic-predominant" and "cortical-predominant" subtypes that resemble previously described MRI-based atrophy subtypes of AD and show corresponding differences in their clinical profiles. By stratifying an independent sample of longitudinally followed prodromal AD patients according to hypometabolic subtype, we could further demonstrate that these subtypes can be detected at a prodromal disease stage and are characterized by differential courses of cognitive decline.

\section{Distinct hypometabolic subtypes amongst patients with AD dementia}

The "typical" subtype included the largest portion of AD dementia cases and was characterized by a typical posterior temporo-parietal pattern of hypometabolism that is commonly linked to $\mathrm{AD}[16,42]$. The "limbic-predominant" subtype had most pronounced hypometabolism in the hippocampus and related medial temporal structures which showed similarities to the MRI-defined medial temporal-dominant atrophy subtypes $[4,33,43$, 44]. Despite these similarities, the "limbic-predominant" subtype in the current study notably differs from previously described medial temporal-dominant atrophy subtypes by showing a more extensive hypometabolic pattern covering widespread limbic areas beyond the medial temporal lobe and including the frontal cortex. A previous study examining FDG-PET patterns in MRIdefined atrophy subtypes also reported pronounced frontal hypometabolism in the medial temporal- 


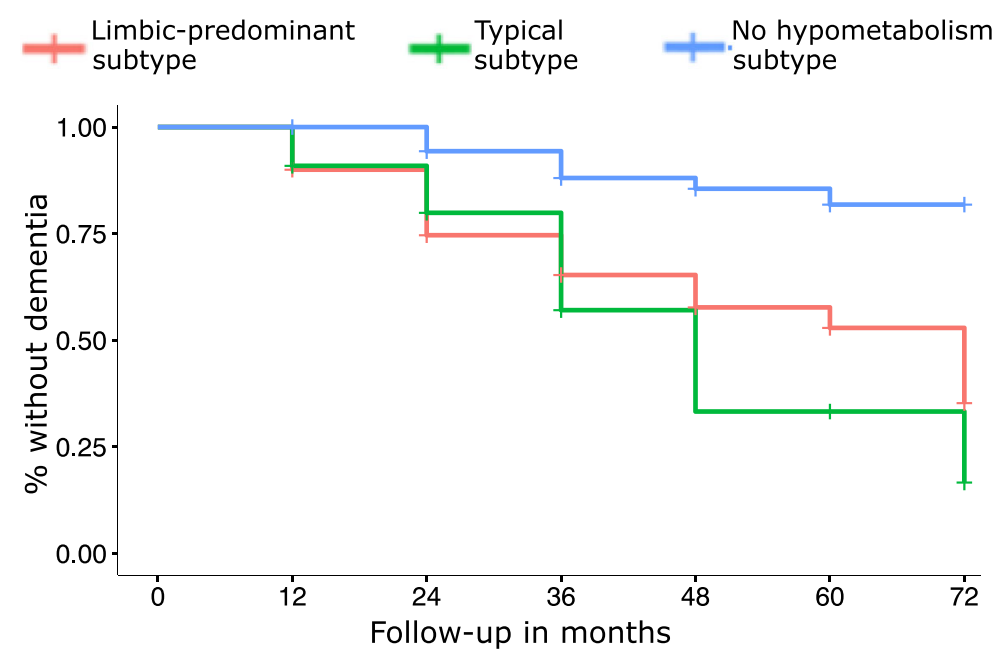

Fig. 3 Kaplan-Meier curves of the time to progression to dementia across subtypes in the prodromal AD group. Kaplan-Meier survival curves indicate proportions of participants within the three prodromal AD subtypes progressing to dementia, operationalized as a change in CDR score from 0.5 to $\geq 1$. Patients who did not progress to dementia within the observation period or did not have follow-up CDR scores were censored

dominant AD atrophy subtype [44]. This could potentially be attributed to differences in the specific pathologic substrate of hypometabolism on FDG-PET and grey matter reductions on MRI as neurodegeneration markers. Specifically, hypometabolism on FDG-PET has been reported to be sensitive to neurodegenerative dysfunction that does not regionally co-localize with neuronal loss as measured by grey matter atrophy on MRI, which may reflect early non-macroscopic neurodegenerative processes or functional changes caused by atrophy in remote but functionally interconnected brain areas $[8,17,45-47]$.

Similarly to previous findings on the MRI-defined medial temporal-predominant atrophy subtype [4, 43], the "limbic-predominant" hypometabolic subtype in the current study was associated with older age and could possibly reflect the effects of comorbid age-related pathologies. For example, Zhang et al. [5] considered that the temporal factor described in their study could be linked to comorbid TDP-43 pathology. Indeed, the hypometabolic pattern of the "limbic-predominant" subtype identified in the present study shows a striking resemblance with a recently described FDG-PET pattern of pathologically confirmed patients with AD dementia with comorbid TDP-43 pathology and hippocampal sclerosis (HS) [48] (specifically, Fig. 3, page 1209 in that study). Corroborating this qualitative visual interpretation, in a complementary post-hoc analysis, we found that the "limbic-predominant" subtype had a significantly higher inferior-to-medial temporal FDG-PET ratio compared to both the "typical" and "cortical-predominant" subtypes (Supplementary Table 6). This ratio has been suggested to reflect the difference between the TDP-43/HS-related pattern and the AD-typical pattern within a simplified metric and has been proposed as an imaging biomarker for comorbid TDP-43/HS in AD [48, 49]. However, it is important to note that any possible involvement of comorbid pathologies in the observed hypometabolic subtypes remains entirely speculative in our in vivo neuroimaging study, and we did not observe any notable differences in the proportion of $\mathrm{AD}$-specific $\mathrm{A} / \mathrm{T}$ biomarker profiles in the limbic-predominant subtype compared to the other subtypes (Supplementary Table 1). Other studies suggested that the medialtemporal subtype could be additionally affected by small vessel disease [4, 50], which would coincide with the numerically highest WMH volume in the "limbic-predominant" subtype in our study. However, this difference did not reach statistical significance in our analysis. Additional neuropathologic examinations as well as mechanistic studies are needed to better understand the exact pathological substrates and neurobiological mechanisms that drive different neurodegeneration subtypes in AD.

The hypometabolic pattern of the "cortical-predominant" subtype was similar to that of the "typical" subtype, but with more extensive involvement of the frontal lobe and largely normal metabolism in the medial temporal lobe. This subtype showed particularly pronounced executive function impairment in addition to the memory deficit. Previously, studies by Collette et al. [11] and Mosconi et al. [12] have also described marked frontal hypometabolism in subsets of patients with AD. On the other side, Ossenkoppele et al. [51] described an autopsy/biomarker-confirmed dysexecutive AD variant, which shows markedly more pronounced impairment in 


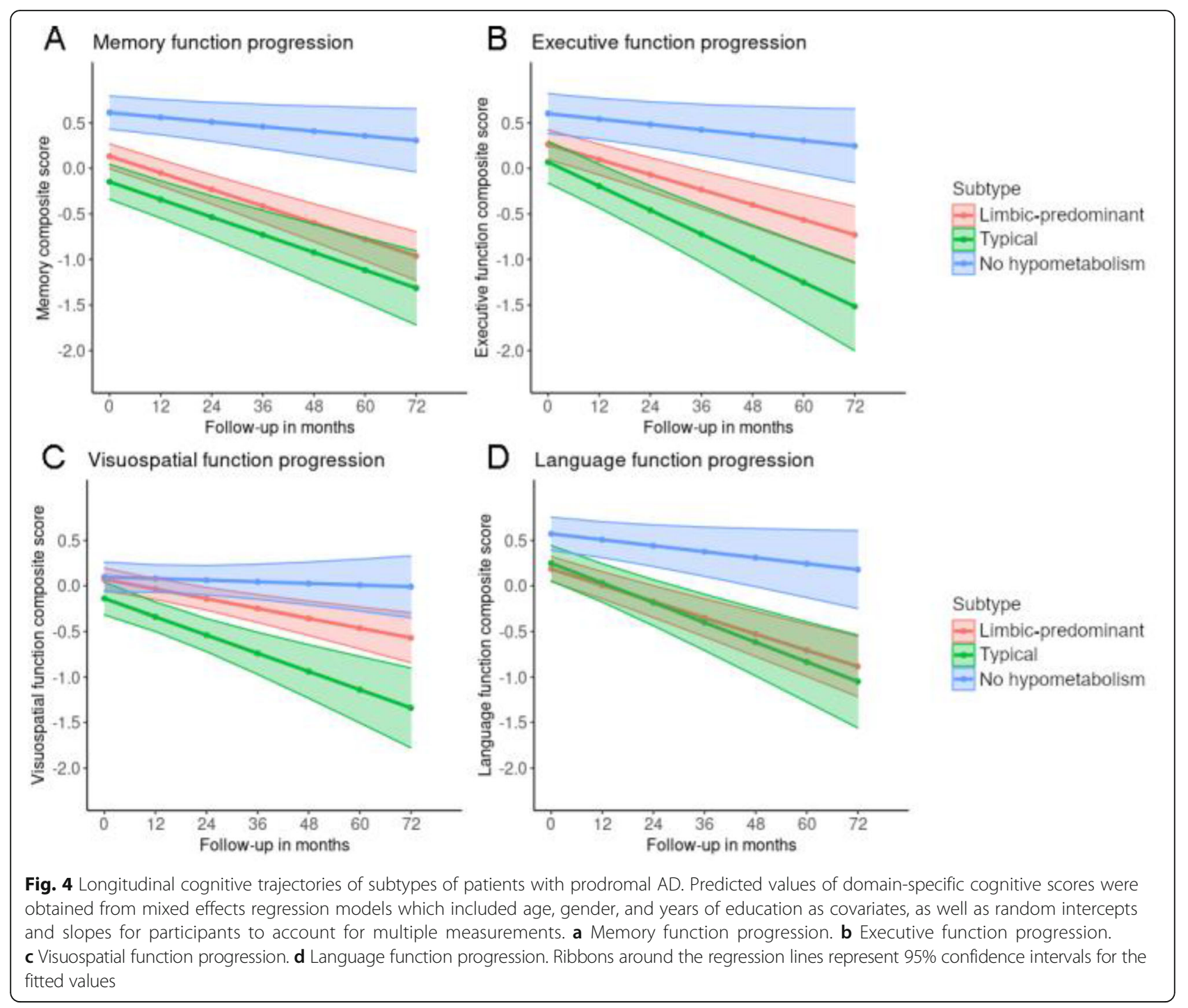

executive function relative to the memory deficit and is characterized by early onset of $\mathrm{AD}$ and a relatively low $A P O E \& 4$ frequency. Similarly, the "cortical-predominant" subtype in the current study also showed the youngest age and lowest percentage of $A P O E$ \&4 carriers amongst $\mathrm{AD}$ subtypes. However, due to the low number of patients in this group, current findings on this subtype require further corroboration.

In accordance with the subtype-defining hypometabolic patterns, we observed a difference between subtypes in the HV:CTV ratio. Specifically, it was the highest for the "cortical-predominant" subtype, intermediate for the "typical" and numerically the lowest for the "limbic-predominant" subtype. The pattern of differences in HV:CTV ratio between FDG-PET subtypes in the current study is comparable to previous findings on AD subtypes based on neuropathological data or MRIbased atrophy patterns. The study by Whitwell et al. [2] examined AD subtypes based on neuropathological examination of distribution of neurofibrillary tangle counts. The ratio between hippocampal and cortical volumes measured on ante-mortem MRI allowed for the best discrimination between these subtypes. In their study, similarly to our results, the typical subtype showed a higher HV:CTV ratio than the limbicpredominant subtype, whereas the hippocampal sparing subtype had the highest value. Furthermore, in the study by Risacher et al. [28], three AD subtypes-hippocampal sparing, limbic predominant and typical $\mathrm{AD}$-were defined based on the HV:CTV ratio. Across these subtypes, a higher HV:CTV ratio was also quantitatively associated with a more pronounced dysexecutive profile, similarly to the differences observed for the ADNI-EF and ADNIDIFF variables between the subtypes in the current study. In a complementary analysis, we could also reproduce this association between $\mathrm{HV}: \mathrm{CTV}$ ratio and 
cognitive profile on a continuous scale (Supplementary Table 7). Thus, across patients, the HV:CTV ratio correlated positively with the ADNI-DIFF variable in both the $\mathrm{AD}$ dementia and prodromal $\mathrm{AD}$ groups, indicating a more pronounced executive function over memory deficit for higher values of this ratio. Therefore, the HV: CTV ratio measured in the current subtypes provides a link between our findings on hypometabolism subtypes and previously characterized AD subtypes based on neuropathological data or MRI-based atrophy patterns. However, there were also differences between the observed hypometabolism subtypes and previously reported atrophy patterns on MRI, such as the aforementioned more extensive involvement of the temporal and frontal areas in the "limbic-predominant" hypometabolism subtype, which might be attributed to the different structural and functional substrates of the respective imaging methods.

\section{Stratification of prodromal AD patients according to hypometabolic subtype}

Stratification of the prodromal AD cohort according to hypometabolic subtypes revealed a considerably sized subgroup of patients with no or only minimal hypometabolism. This "no hypometabolism" subtype also showed lower levels of $A \beta$ biomarker burden, was less cognitively impaired and had a lower risk of progressing to dementia compared to the other subtypes. While this may indicate that this subtype may be enriched for patients with only incidental amyloidosis, the comparably high proportion of concomitant tau biomarker positivity in this group (Supplementary Table 1) would rather argue against this possibility.

Previous studies using visual classification of FDG-PET scans had also described subsets of patients with MCI without evidence of regional hypometabolism $[12,14]$. In the study by Cerami et al. [14], 31\% of participants with MCI showed normal brain metabolism, although the large majority of these also had a negative amyloid biomarker finding. However, MRI-based subtyping studies have also consistently identified subsets of patients with AD dementia with no or only minimal atrophy, and this subtype was particularly prevalent amongst patients with prodromal $\mathrm{AD}[4,43,52]$. Interestingly, in our study, we observed a similar "minimal" hypometabolism subtype in the AD dementia group when using a higher clustering solution (see Supplementary Figure 2). However, since we established the best distinguishable AD subtypes using an objective hierarchical clustering cutoff as suggested by the Davies-Bouldin and the silhouette criteria, we did not further characterize this "minimal" subtype in our study. Nevertheless, our findings underline the importance of accounting for the considerably sized subgroup of patients with prodromal $\mathrm{AD}$ without evidence of regional hypometabolism when characterizing heterogeneity of hypometabolism patterns in this population.

Only three participants with prodromal AD were classified into the "cortical-predominant" subtype and could thus not be further analysed in our study. One potential explanation for this low prevalence could be that this hypometabolic subtype is characterized by more pronounced executive function deficits than memory deficits from its prodromal stage on, so that these patients would be underrepresented in an MCI cohort screened for memory deficits such as the ADNI cohort.

The "limbic-predominant" and "typical" subtypes classified in the prodromal AD sample demonstrated similar subtype characteristics as in the AD dementia sample. Thus, the "limbic-predominant" subtype also had older age, numerically higher WMH volume and the most severe degree of hippocampus atrophy. Hence, current results confirm previous findings that the heterogeneity evident in patients with $\mathrm{AD}$ dementia can also be observed at the prodromal stage of the disease [4, 5]. Interestingly, although the "limbic-predominant" and "typical" subtypes had a comparable risk of progressing to dementia, the "limbic-predominant" subtype showed a more memory-selective cognitive decline compared to the "typical" subtype, paralleling the cross-sectional subtype differences in cognitive profiles observed in the $A D$ dementia cohort. This finding is notable, because these subsets of patients with prodromal AD did not show significant differences in the respective cognitive functions at baseline. This indicates that subtype classification of FDG-PET patterns may provide additional information for predicting future cognitive decline that is not contained in neuropsychological assessments.

Our current findings on subtype-specific trajectories of cognitive decline are largely consistent with previous findings on differences in dementia risk and domainspecific cognitive decline between atrophy subtypes based on MRI data. For example, the studies by Ten Kate et al. [4] and Dong et al. [5] both only found a significantly lower risk for progression to dementia in the no/minimal atrophy subtype, and while the other atrophy subtypes showed similar overall risk for progression to dementia, they differed significantly in the relative decline in specific cognitive domains.

A previous study by Morbelli et al. [53] used classical voxel-wise analyses of FDG-PET images in a group of prodromal AD patients to determine a "prognostic pattern" of regional brain hypometabolism that best correlated with time to conversion to dementia. Interestingly, this "prognostic pattern" was found to be considerably different from the AD-typical "diagnostic pattern" of hypometabolism as determined by contrasting $\mathrm{AD}$ patients with healthy controls. In our current study, we used a data-driven approach to demonstrate that this 
typical group-averaged "diagnostic pattern" can be decomposed into different regional subtypes corresponding to distinct subgroups of AD patients. It is likely that these different FDG-PET subtypes also correspond to different "prognostic patterns" that best predict time to dementia conversion in the respective subgroups of prodromal AD patients.

\section{Strengths and limitations}

One conceptual strength of the current study is that we only included patients with biomarker evidence of $A \beta$ pathology. Moreover, we used the identified hypometabolism subtypes in patients with AD dementia to classify an independent dataset of $A \beta$-positive patients with $\mathrm{MCI}$ and assess clinical and biomarker characteristics of these subtypes at a prodromal disease stage.

As with other unsupervised subtyping studies, a principal limitation of the current study is that the employed clustering methodology cannot naturally distinguish between subtypes and different disease stages. We aimed to mitigate the effect of differing disease stages by normalizing the individual FDG-PET profiles to their global signal before clustering, so that the cluster assignations were primarily driven by relative regional metabolic differences instead of global differences accompanying disease progression. Global signal scaling is a commonly used method to control neuroimaging clustering analyses for individual differences in disease severity, and analogous approaches have been used in previous FDGPET subtyping studies in other neurodegenerative dementias [32, 34], as well as in MRI-based subtyping studies of neurodegeneration heterogeneity in AD (see, e.g. [3] for a recent review of this literature). We also note that the hypometabolic characteristics of the identified subtypes would not be consistent with the notion of merely reflecting variability in disease severity. As an example, the interpretation that the "limbic-predominant" subtype would reflect an earlier stage of the "typical" subtype cannot be easily reconciled with the observation of more severe medial temporal hypometabolism in the "limbic-predominant" subtype. A similar argument also applies to the comparison between the "typical" and "cortical-predominant" subtypes (also see Supplementary Figure 3 for a direct voxel-wise comparison of regional hypometabolic differences between the subtypes).

The contribution of disease stage to observed subtype phenotypes has been addressed in a recent MRI-based subtyping study by Young et al. [54], which proposed an analytical approach combining clustering with eventbased modelling to assess subtypes and their respective stage progressions at the same time. However, this approach still relies on extrapolations from cross-sectional data. Future research on neurodegeneration subtypes in $\mathrm{AD}$ will benefit from longitudinal imaging assessments allowing to directly characterize disease progression within subtypes and to determine the possibility of conversion between them.

\section{Conclusion}

In the current study, we used a systematic data-driven approach for characterizing differential neurodegeneration subtypes in $\mathrm{AD}$ as reflected by hypometabolism patterns on FDG-PET. The hypometabolic subtypes were associated with differential clinical and biomarker profiles, as well as with differences in clinical trajectories over time. These findings complement recent research efforts on characterizing distinct atrophy subtypes in $\mathrm{AD}$ using structural MRI data. Due to the reportedly higher sensitivity of FDG-PET for early neurodegenerative changes, the described hypometabolic subtypes may provide a sensitive tool for early detection and characterization of AD-related neurodegeneration variants at prodromal disease stages, which may have important implications for improving timely and differentiated prognosis in non-demented individuals with biomarker evidence of AD.

\section{Supplementary Information}

The online version contains supplementary material available at https://doi. org/10.1186/s13195-021-00785-9.

Additional file 1: Supplementary Table 1. A/T/N profiles across AD and prodromal AD subtypes. Supplementary Table 2. Structures defined in the Harvard-Oxford atlas that were used to measure the composite cortical volume. Supplementary Table 3. Hazard ratios for progression of subtypes of patients with prodromal AD to dementia. Supplementary Table 4. Mixed effects regression models of longitudinal cognitive decline across subtypes in the prodromal AD group; "no hypometabolism" subtype as reference. Supplementary Table 5. Mixed effects regression models of longitudinal cognitive decline across subtypes in the prodromal AD group; "limbic-predominant" subtype as reference. Supplementary Table 6. Comparisons between AD subtypes with respect to the ratio of inferior to medial temporal metabolism assessed with FDG-PET. Supplementary Table 7. Correlations between the HV: CTV ratio and cognitive measures in the $A D$ and in the prodromal AD groups. Supplementary Table 8. Missing values (at baseline) for demographic, clinical and biomarker characteristics in the AD dementia and prodromal AD groups. Supplementary Figure 1. Determination of optimal clustering cutoff by objective criteria. Supplementary Figure 2. Hierarchical clustering dendrogram and hypometabolic FDG-PET patterns of resulting AD subtypes at higher cluster solutions. Supplementary

Figure 3. Comparisons of hypometabolic FDG-PET patterns of subtypes of patients with AD. Supplementary Figure 4. Hypometabolic FDG-PET patterns of subtypes of patients with prodromal AD with adjusted scale.

\section{Abbreviations}

AD: Alzheimer's disease; ADNI: Alzheimer's Disease Neuroimaging Initiative; ADNI-DIFF: Difference between ADNI-MEM and ADNI-EF; ADNI-EF: ADNI composite cognitive score for executive function; ADNI-MEM: ADNI composite cognitive score for memory function; APOE: Apolipoprotein E; A $\beta$ : Beta-amyloid; CDR: Clinical Dementia Rating; CN: Cognitively normal; CSF: Cerebrospinal fluid; CTV: Cortical composite grey matter volume scaled to total intracranial volume; FDG: Fluorodeoxyglucose; FDR: False discovery rate; GM: Grey matter; HV: Hippocampal grey matter volume scaled to total intracranial volume; MCI: Mild cognitive impairment; MMSE: Mini-Mental State Examination; MRI: Magnetic resonance imaging; PET: Positron emission 
tomography; SUVR: Standard uptake value ratio; TIV: Total intracranial volume; WM: White matter; WMH: White matter hyperintensity volume

\section{Acknowledgements}

The authors would like to thank the Swedish Research Council (VR)

\section{Authors' contributions}

FL, SJT and MJG designed and conceptualized the study. FL, SJT, MJG and MD analysed the data. FL, SJT, MJG and DF drafted the manuscript for intellectual content. All authors interpreted the data, revised the manuscript for intellectual content and read and approved the final manuscript.

\section{Funding}

Michel J. Grothe is supported by the "Miguel Servet" program [CP19/00031] and a research grant [PI20/00613] of the Instituto de Salud Carlos III-Fondo Europeo de Desarrollo Regional (ISCIII-FEDER).

Data collection and sharing for this project was funded by the Alzheimer's Disease Neuroimaging Initiative (ADNI) (National Institutes of Health Grant U01 AG024904) and DOD ADNI (Department of Defense award number W81XWH-12-2-0012). ADNI is funded by the National Institute on Aging and the National Institute of Biomedical Imaging and Bioengineering and through generous contributions from the following: AbbVie, Alzheimer's Association; Alzheimer's Drug Discovery Foundation; Araclon Biotech; BioClinica, Inc.; Biogen; Bristol-Myers Squibb Company; CereSpir, Inc.; Cogstate; Eisai Inc.; Elan Pharmaceuticals, Inc.; Eli Lilly and Company; Eurolmmun; F. Hoffmann-La Roche Ltd. and its affiliated company Genentech, Inc.; Fujirebio; GE Healthcare; IXICO Ltd.; Janssen Alzheimer Immunotherapy Research \& Development, LLC.; Johnson \& Johnson Pharmaceutical Research \& Development LLC.; Lumosity; Lundbeck; Merck \& Co., Inc.; Meso Scale Diagnostics, LLC.; NeuroRx Research; Neurotrack Technologies; Novartis Pharmaceuticals Corporation; Pfizer Inc.; Piramal Imaging; Servier; Takeda Pharmaceutical Company; and Transition Therapeutics. The Canadian Institutes of Health Research is providing funds to support ADNI clinical sites in Canada. Private sector contributions are facilitated by the Foundation for the National Institutes of Health (www.fnih. org). The grantee organization is the Northern California Institute for Research and Education, and the study is coordinated by the Alzheimer's Therapeutic Research Institute at the University of Southern California. ADNI data are disseminated by the Laboratory for Neuro Imaging at the University of Southern California. Open Access funding enabled and organized by Projekt DEAL.

\section{Availability of data and materials}

Data analysed in this study were acquired from the Alzheimer's Disease Neuroimaging Initiative (ADNI) database (http://adni.loni.usc.edu). ADNI data are shared in a de-identified form and without embargo subject to a review of a data use application by the ADNI Data Sharing and Publications Committee. For further information, please refer to the ADNI website (http:// adni.loni.usc.edu/data-samples/access-data/).

\section{Ethics approval and consent to participate}

Data collection and sharing in ADNI was approved by the Institutional Review Board of each participating institution. All procedures involving human participants were in accordance with the ethical standards of the 1964 Helsinki Declaration and its later amendments. Written informed consent was obtained from all ADNI participants and/or authorized representatives before any protocol-specific procedures were carried out.

\section{Consent for publication}

Not applicable.

\section{Competing interests}

SJT participated in the scientific advisory boards of Roche Pharma AG and MSD and received lecture fees from Roche and MSD. MJG, FL, CL, MD, EW and RB declare that they have no competing interests.

\section{Author details}

'German Center for Neurodegenerative Diseases (DZNE), Rostock/Greifswald, Rostock, Germany. ${ }^{2}$ Division of Clinical Geriatrics, Department of Neurobiology, Care Sciences and Society, Center for Alzheimer Research, Karolinska Institutet, Stockholm, Sweden. ${ }^{3}$ Department of Nuclear Medicine, Charité - Universitätsmedizin Berlin, Corporate Member of Freie Universität
Berlin, Humboldt-Universität zu Berlin and Berlin Institute of Health, Berlin, Germany. ${ }^{4}$ German Center for Neurodegenerative Diseases (DZNE), Dresden, Germany. ${ }^{5}$ Department of Neuroimaging, Centre for Neuroimaging Sciences, Institute of Psychiatry, Psychology and Neuroscience, King's College London, London, UK. ${ }^{6}$ Department of Diagnostic and Interventional Radiology and Nuclear Medicine, University Medical Center Hamburg-Eppendorf, Hamburg, Germany. ${ }^{7}$ Department of Psychosomatic Medicine, University of Rostock, Rostock, Germany. ${ }^{8}$ Unidad de Trastornos del Movimiento, Servicio de Neurología y Neurofisiología Clínica, Instituto de Biomedicina de Sevilla, Hospital Universitario Virgen del Rocío/CSIC/Universidad de Sevilla, Avda. Manuel Siurot, s/n, 41013 Sevilla, Spain.

Received: 29 October 2020 Accepted: 3 February 2021 Published online: 19 February 2021

\section{References}

1. Murray ME, Graff-Radford NR, Ross OA, Petersen RC, Duara R, Dickson DW. Neuropathologically defined subtypes of Alzheimer's disease with distinct clinical characteristics: a retrospective study. Lancet Neurol. 2011:10(9):785-96.

2. Whitwell JL, Dickson DW, Murray ME, Weigand SD, Tosakulwong N, Senjem $\mathrm{ML}$, et al. Neuroimaging correlates of pathologically defined subtypes of Alzheimer's disease: a case-control study. Lancet Neurol. 2012;11(10):868-77.

3. Habes M, Grothe MJ, Tunc B, McMillan C, Wolk DA, Davatzikos C. Disentangling heterogeneity in Alzheimer's disease and related dementias using data-driven methods. Biol Psychiatry. 2020;88(1):70-82. PMID: 32201044 .

4. Ten Kate M, Dicks E, Visser PJ, van der Flier WM, Teunissen CE, Barkhof F, et al. Atrophy subtypes in prodromal Alzheimer's disease are associated with cognitive decline. Brain. 2018;141(12):3443-56.

5. Zhang X, Mormino EC, Sun N, Sperling RA, Sabuncu MR, Yeo BT, et al. Bayesian model reveals latent atrophy factors with dissociable cognitive trajectories in Alzheimer's disease. Proc Natl Acad Sci U S A. 2016;113(42): E6535-E44.

6. Jack CR Jr, Bennett DA, Blennow K, Carrillo MC, Dunn B, Haeberlein SB, et al. NIA-AA Research Framework: toward a biological definition of Alzheimer's disease. Alzheimers Dement. 2018;14(4):535-62.

7. Nobili F, Arbizu J, Bouwman F, Drzezga A, Agosta F, Nestor P, et al. European Association of Nuclear Medicine and European Academy of Neurology recommendations for the use of brain ${ }^{18} \mathrm{~F}$-fluorodeoxyglucose positron emission tomography in neurodegenerative cognitive impairment and dementia: Delphi consensus. Eur J Neurol. 2018;25(10):1201-17.

8. Kljajevic V, Grothe MJ, Ewers M, Teipel S. Alzheimer's disease neuroimaging 1. Distinct pattern of hypometabolism and atrophy in preclinical and predementia Alzheimer's disease. Neurobiol Aging. 2014;35(9):1973-81.

9. Perani D. FDG-PET and amyloid-PET imaging: the diverging paths. Curr Opin Neurol. 2014;27(4):405-13.

10. Shaffer JL, Petrella JR, Sheldon FC, Choudhury KR, Calhoun VD, Coleman RE, et al. Predicting cognitive decline in subjects at risk for Alzheimer disease by using combined cerebrospinal fluid, MR imaging, and PET biomarkers. Radiology. 2013;266(2):583-91.

11. Collette F, Van der Linden M, Delrue G, Salmon E. Frontal hypometabolism does not explain inhibitory dysfunction in Alzheimer disease. Alzheimer Dis Assoc Disord. 2002;16(4):228-38.

12. Mosconi L, Tsui WH, Herholz K, Pupi A, Drzezga A, Lucignani G, et al. Multicenter standardized 18F-FDG PET diagnosis of mild cognitive impairment, Alzheimer's disease, and other dementias. J Nucl Med. 2008; 49(3):390-8

13. Meyer F, Wehenkel M, Phillips C, Geurts P, Hustinx R, Bernard C, et al. Characterization of a temporoparietal junction subtype of Alzheimer's disease. Hum Brain Mapp. 2019;40(14):4279-86.

14. Cerami C, Della Rosa PA, Magnani G, Santangelo R, Marcone A, Cappa SF, et al. Brain metabolic maps in mild cognitive impairment predict heterogeneity of progression to dementia. Neurolmage Clin. 2015;7:187-94.

15. Perani D, Cerami C, Caminiti SP, Santangelo R, Coppi E, Ferrari L, et al. Cross-validation of biomarkers for the early differential diagnosis and prognosis of dementia in a clinical setting. Eur J Nucl Med Mol Imaging. 2016:43(3):499-508.

16. Nestor PJ, Altomare D, Festari C, Drzezga A, Rivolta J, Walker Z, et al. Clinical utility of FDG-PET for the differential diagnosis among the main forms of dementia. Eur J Nucl Med Mol Imaging. 2018;45(9):1509-25. 
17. Teipel S, Grothe MJ, Alzheimer s Disease Neuroimaging I. Does posterior cingulate hypometabolism result from disconnection or local pathology across preclinical and clinical stages of Alzheimer's disease? Eur J Nucl Med Mol Imaging. 2016;43(3):526-36.

18. Crane PK, Carle A, Gibbons LE, Insel P, Mackin RS, Gross A, et al. Development and assessment of a composite score for memory in the Alzheimer's Disease Neuroimaging Initiative (ADNI). Brain Imaging Behav. 2012;6(4):502-16.

19. Gibbons LE, Carle AC, Mackin RS, Harvey D, Mukherjee S, Insel P, et al. A composite score for executive functioning, validated in Alzheimer's Disease Neuroimaging Initiative (ADNI) participants with baseline mild cognitive impairment. Brain Imaging Behav. 2012;6(4):517-27.

20. Choi SE, Mukherjee S, Gibbons LE, Sanders RE, Jones RN, Tommet D, et al. Development and validation of language and visuospatial composite scores in ADNI. Alzheimers Dement (N Y). 2020;6(1):e12072.

21. Landau SM, Thomas BA, Thurfjell L, Schmidt M, Margolin R, Mintun M, et al. Amyloid PET imaging in Alzheimer's disease: a comparison of three radiotracers. Eur J Nucl Med Mol Imaging. 2014;41(7):1398-407.

22. Hansson O, Seibyl J, Stomrud E, Zetterberg H, Trojanowski JQ, Bittner T, et al. CSF biomarkers of Alzheimer's disease concord with amyloid-beta PET and predict clinical progression: a study of fully automated immunoassays in BioFINDER and ADNI cohorts. Alzheimers Dement. 2018;14(11):1470-81.

23. Salvado G, Molinuevo JL, Brugulat-Serrat A, Falcon C, Grau-Rivera O, SuarezCalvet $\mathrm{M}$, et al. Centiloid cut-off values for optimal agreement between PET and CSF core AD biomarkers. Alzheimers Res Ther. 2019;11(1):27.

24. Saykin AJ, Shen L, Foroud TM, Potkin SG, Swaminathan S, Kim S, et al. Alzheimer's Disease Neuroimaging Initiative biomarkers as quantitative phenotypes: genetics core aims, progress, and plans. Alzheimers Dement. 2010;6(3):265-73.

25. Grothe MJ, Villeneuve S, Dyrba M, Bartres-Faz D, Wirth M, Alzheimer's Disease Neuroimaging I. Multimodal characterization of older APOE2 carriers reveals selective reduction of amyloid load. Neurology. 2017;88(6):569-76.

26. Wolf D, Bocchetta M, Preboske GM, Boccardi M, Grothe MJ, Alzheimer's Disease Neuroimaging I. Reference standard space hippocampus labels according to the European Alzheimer's Disease Consortium-Alzheimer's Disease Neuroimaging Initiative harmonized protocol: utility in automated volumetry. Alzheimers Dement. 2017;13(8):893-902.

27. Desikan RS, Segonne F, Fischl B, Quinn BT, Dickerson BC, Blacker D, et al. An automated labeling system for subdividing the human cerebral cortex on MRI scans into gyral based regions of interest. Neuroimage. 2006;31(3):968-80.

28. Risacher SL, Anderson WH, Charil A, Castelluccio PF, Shcherbinin S, Saykin AJ, et al. Alzheimer disease brain atrophy subtypes are associated with cognition and rate of decline. Neurology. 2017;89(21):2176-86.

29. Schwarz C, Fletcher E, DeCarli C, Carmichael O. Fully-automated white matter hyperintensity detection with anatomical prior knowledge and without FLAIR. Inf Process Med Imaging. 2009;21:239-51.

30. DeCarli C, Fletcher E, Ramey V, Harvey D, Jagust WJ. Anatomical mapping of white matter hyperintensities $(\mathrm{WMH})$ : exploring the relationships between periventricular $\mathrm{WMH}$, deep $\mathrm{WMH}$, and total $\mathrm{WMH}$ burden. Stroke. 2005;36(1):50-5.

31. Lange C, Suppa P, Frings L, Brenner W, Spies L, Buchert R. Optimization of statistical single subject analysis of brain FDG PET for the prognosis of mild cognitive impairment-to-Alzheimer's disease conversion. J Alzheimers Dis. 2016:49(4):945-59.

32. Matias-Guiu JA, Diaz-Alvarez J, Ayala JL, Risco-Martin JL, Moreno-Ramos T, Pytel $V$, et al. Clustering analysis of FDG-PET imaging in primary progressive aphasia. Front Aging Neurosci. 2018;10:230.

33. Noh Y, Jeon S, Lee JM, Seo SW, Kim GH, Cho H, et al. Anatomical heterogeneity of Alzheimer disease: based on cortical thickness on MRIs. Neurology. 2014;83(21):1936-44.

34. Cerami C, Dodich A, Lettieri G, lannaccone S, Magnani G, Marcone A, et al. Different FDG-PET metabolic patterns at single-subject level in the behavioral variant of fronto-temporal dementia. Cortex. 2016;83:101-12.

35. Davies DL, Bouldin DW. A cluster separation measure. IEEE Trans Pattern Anal Mach Intell. 1979;1(2):224-7.

36. Rousseeuw PJ. Silhouettes: a graphical aid to the interpretation and validation of cluster analysis. J Comput Appl Math. 1987;20:53-65.

37. Dukart J, Mueller K, Horstmann A, Vogt B, Frisch S, Barthel H, et al. Differential effects of global and cerebellar normalization on detection and differentiation of dementia in FDG-PET studies. Neurolmage. 2010;49(2):1490-5.
38. Yakushev I, Landvogt C, Buchholz HG, Fellgiebel A, Hammers A, Scheurich A, et al. Choice of reference area in studies of Alzheimer's disease using positron emission tomography with fluorodeoxyglucose-F18. Psychiatry Res. 2008;164(2):143-53.

39. Benjamini $Y$, Yekutieli $D$. The control of the false discovery rate in multiple testing under dependency. Ann Stat. 2001;29(4):1165-88.

40. Teipel SJ, Dyrba M, Chiesa PA, Sakr F, Jelistratova I, Lista S, et al. In vivo staging of regional amyloid deposition predicts functional conversion in the preclinical and prodromal phases of Alzheimer's disease. Neurobiol Aging. 2020;93:98-108

41. Ray NJ, Bradburn S, Murgatroyd C, Toseeb U, Mir P, Kountouriotis GK, et al. In vivo cholinergic basal forebrain atrophy predicts cognitive decline in de novo Parkinson's disease. Brain. 2018;141(1):165-76.

42. Mosconi $\mathrm{L}$. Brain glucose metabolism in the early and specific diagnosis of Alzheimer's disease. FDG-PET studies in MCl and AD. Eur J Nucl Med Mol Imaging. 2005;32(4):486-510.

43. Poulakis K, Pereira JB, Mecocci P, Vellas B, Tsolaki M, Kloszewska I, et al. Heterogeneous patterns of brain atrophy in Alzheimer's disease. Neurobiol Aging. 2018;65:98-108

44. Hwang J, Kim CM, Jeon S, Lee JM, Hong YJ, Roh JH, et al. Prediction of Alzheimer's disease pathophysiology based on cortical thickness patterns. Alzheimers Dement (Amst). 2016:2:58-67.

45. Klupp E, Grimmer T, Tahmasian M, Sorg C, Yakushev I, Yousefi BH, et al. Prefrontal hypometabolism in Alzheimer disease is related to longitudinal amyloid accumulation in remote brain regions. J Nucl Med. 2015:56(3):399-404.

46. La Joie R, Landeau B, Perrotin A, Bejanin A, Egret S, Pélerin A, et al. Intrinsic connectivity identifies the hippocampus as a main crossroad between Alzheimer's and semantic dementia-targeted networks. Neuron. 2014;81(6): 1417-28.

47. Villain N, Fouquet M, Baron JC, Mezenge F, Landeau B, de La Sayette V, et al. Sequential relationships between grey matter and white matter atrophy and brain metabolic abnormalities in early Alzheimer's disease. Brain. 2010;133(11):3301-14.

48. Botha H, Mantyh WG, Murray ME, Knopman DS, Przybelski SA, Wiste HJ, et al. FDG-PET in tau-negative amnestic dementia resembles that of autopsy-proven hippocampal sclerosis. Brain. 2018;141(4):1201-17.

49. Buciuc M, Botha H, Murray ME, Schwarz CG, Senjem ML, Jones DT, et al. Utility of FDG-PET in diagnosis of Alzheimer-related TDP-43 proteinopathy. Neurology. 2020;95(1):e23-34.

50. Ferreira D, Shams S, Cavallin L, Viitanen M, Martola J, Granberg T, et al. The contribution of small vessel disease to subtypes of Alzheimer's disease: a study on cerebrospinal fluid and imaging biomarkers. Neurobiol Aging. 2018;70:18-29.

51. Ossenkoppele R, Pijnenburg YA, Perry DC, Cohn-Sheehy BI, Scheltens NM, Vogel JW, et al. The behavioural/dysexecutive variant of Alzheimer's disease: clinical, neuroimaging and pathological features. Brain. 2015:138(Pt 9):2732-49.

52. Dong A, Toledo JB, Honnorat N, Doshi J, Varol E, Sotiras A, et al. Heterogeneity of neuroanatomical patterns in prodromal Alzheimer's disease: links to cognition, progression and biomarkers. Brain. 2017;140(3): 735-47.

53. Morbelli S, Bauckneht M, Arnaldi D, Picco A, Pardini M, Brugnolo A, et al. 18F-FDG PET diagnostic and prognostic patterns do not overlap in Alzheimer's disease (AD) patients at the mild cognitive impairment (MCl) stage. Eur J Nucl Med Mol Imaging. 2017:44(12):2073-83.

54. Young AL, Marinescu RV, Oxtoby NP, Bocchetta M, Yong K, Firth NC, et al. Uncovering the heterogeneity and temporal complexity of neurodegenerative diseases with subtype and stage inference. Nat Commun. 2018;9(1):4273

\section{Publisher's Note}

Springer Nature remains neutral with regard to jurisdictional claims in published maps and institutional affiliations. 